\title{
Discovery of Selective Pituitary Adenylate Cyclase 1 Receptor (PAC1R) Antagonist Peptides Potent in Maxadilan/PACAP38 Induced Increase in Blood Flow Pharmacodynamics Model
}

Essa Hu ${ }^{*, \S}$ Fang-Tsao Hong, ${ }^{\S}$ Jennifer Aral, ${ }^{\S}$ Jason Long, ${ }^{\S}$ Derek Piper, ${ }^{\ddagger}$ Leszek Poppe, ${ }^{\S}$ Kristin L. Andrews, ${ }^{\S}$ Narender Gavva, ${ }^{\square}$ Todd Hager," Carl Davis, "Hongyan Li," Philip Wong," Licheng Shi, Dawn X.D. Zhu, ${ }^{\square}$ Sonya G. Lehto, ${ }^{1}$ Cen Xu, Les P. Miranda ${ }^{\S}$

${ }^{\S}$ Therapeutic Discovery, ${ }^{\square}$ Neuroscience Research, and "Pharmacokinetics and Drug Metabolism, Amgen Research, Amgen Inc., One Amgen Center Drive, Thousand Oaks, California, 91320, United States

${ }^{*}$ Therapeutic Discovery, Amgen Research, Amgen Inc., 1120 Veterans Blvd, South San Francisco, California 94080, United States

\section{Supporting Information}

\section{Table of Contents:}

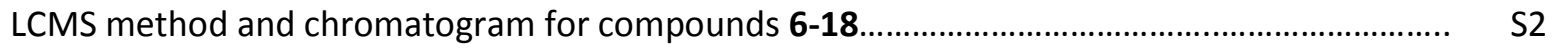

Characterization data table for compounds 6 -18...................................................................... S15

Crystal collection data and structure refinement statistics for co-crystal structure of the PAC1R extracellular domain in complex with peptide 18

Relevant SAR from alanine scanning, Aib scanning, and single residue deletion scanning.......... S18

Additional information on commercial M65 tested and potency comparison table......

S18 
Chromatography Method: Max-RP column, 2.5 um, $50 \times 2 \mathrm{~mm}$; eluent $A=\mathrm{H}_{2} \mathrm{O}(0.1 \%$ TFA), $B=A C N$ in $\mathrm{H}_{2} \mathrm{O}$ (0.1\% TFA), 5-50\%B over $10 \mathrm{~min} @ 750 \mathrm{uL} / \mathrm{min}$

LCMS chromatogram for compound 6
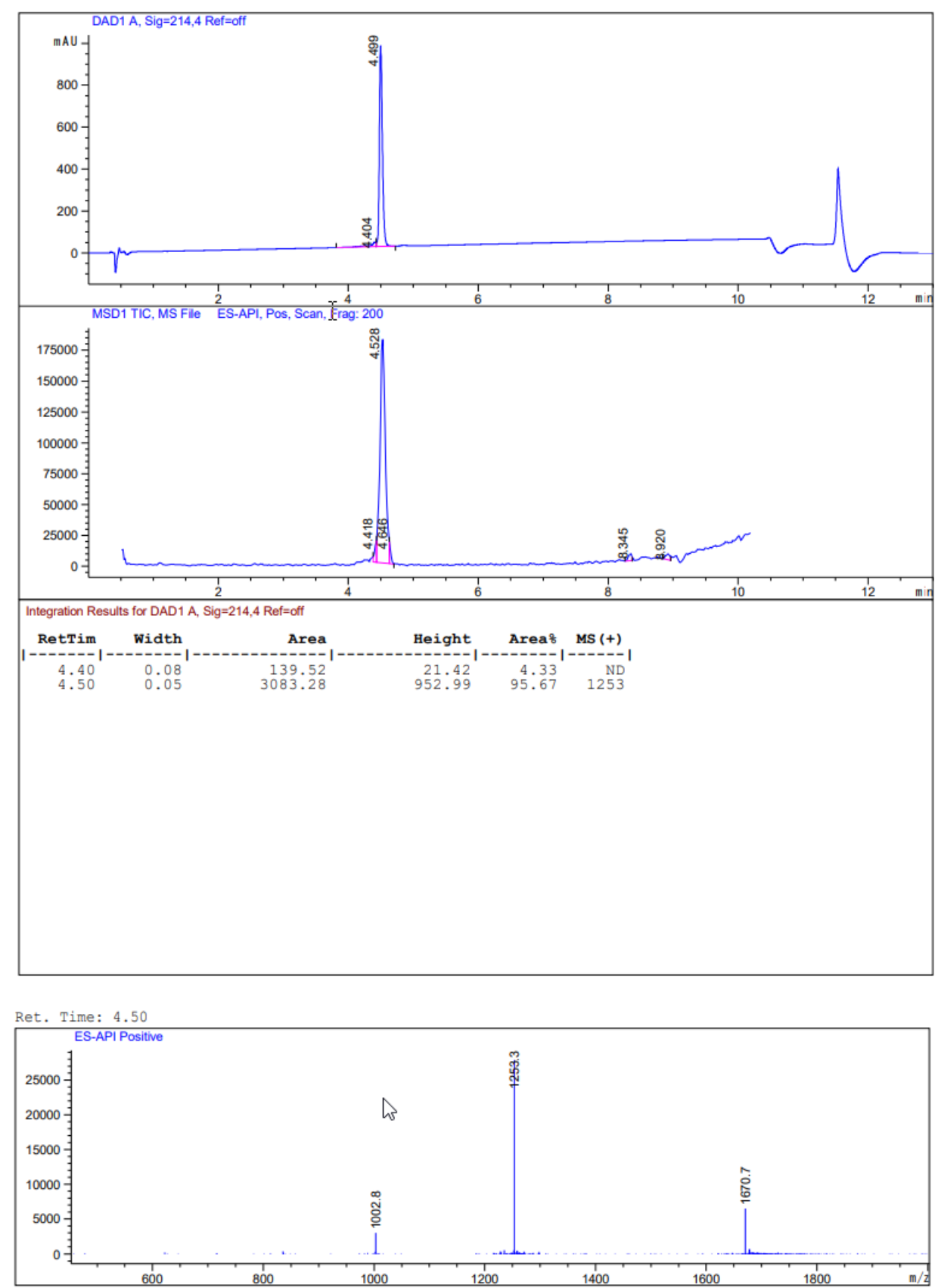
LCMS chromatogram for compound 7
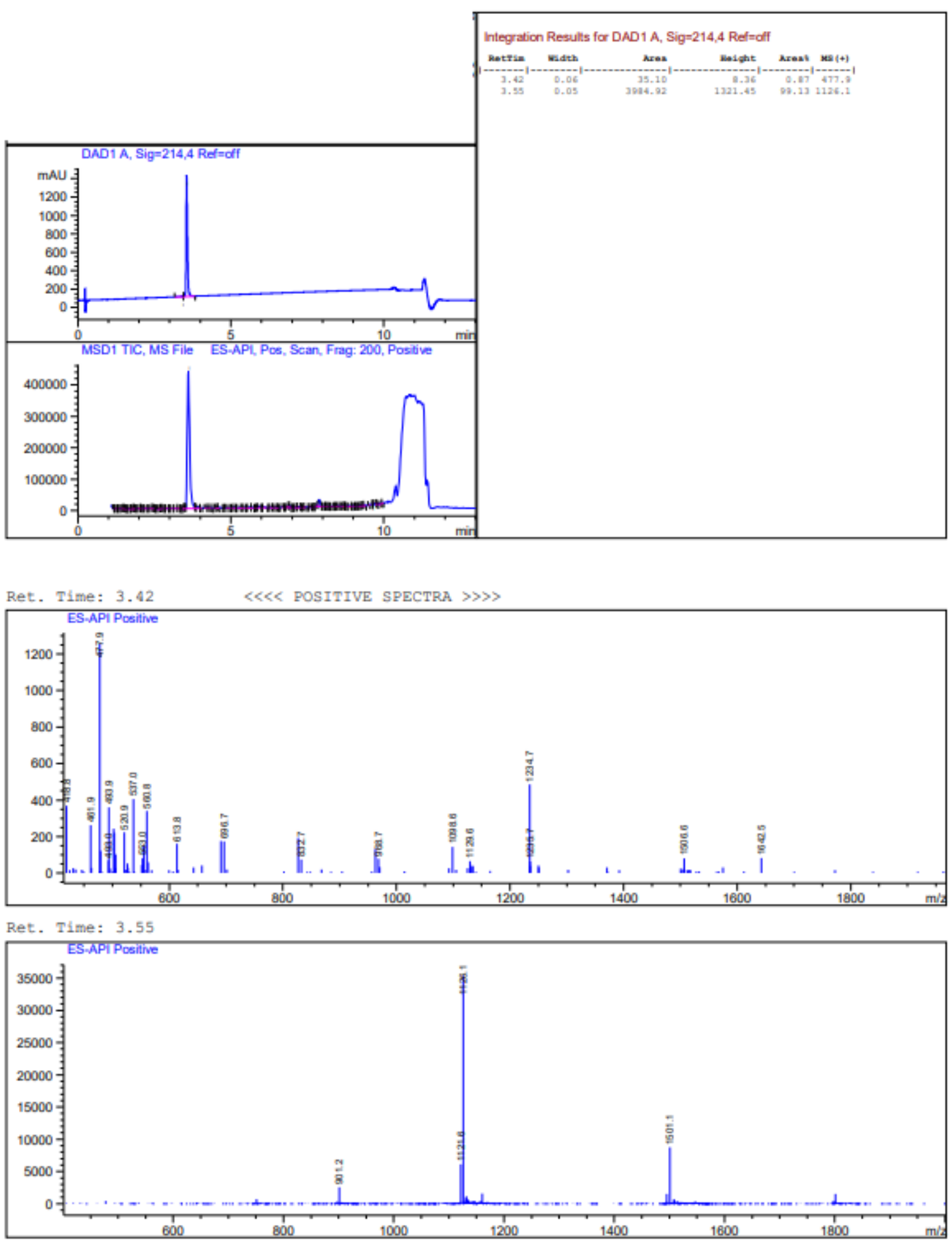
LCMS chromatogram for compound 8
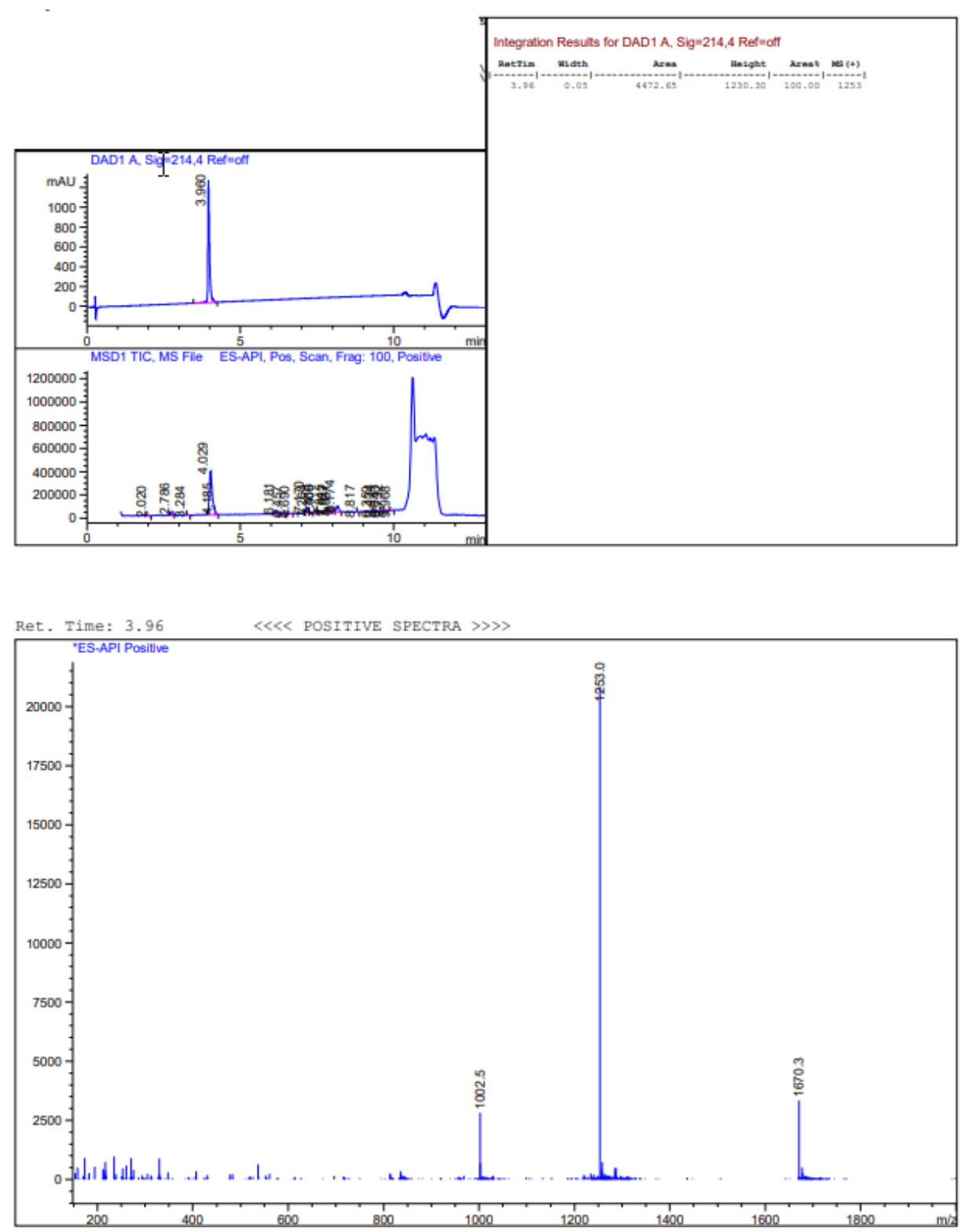
LCMS chromatogram for compound 9
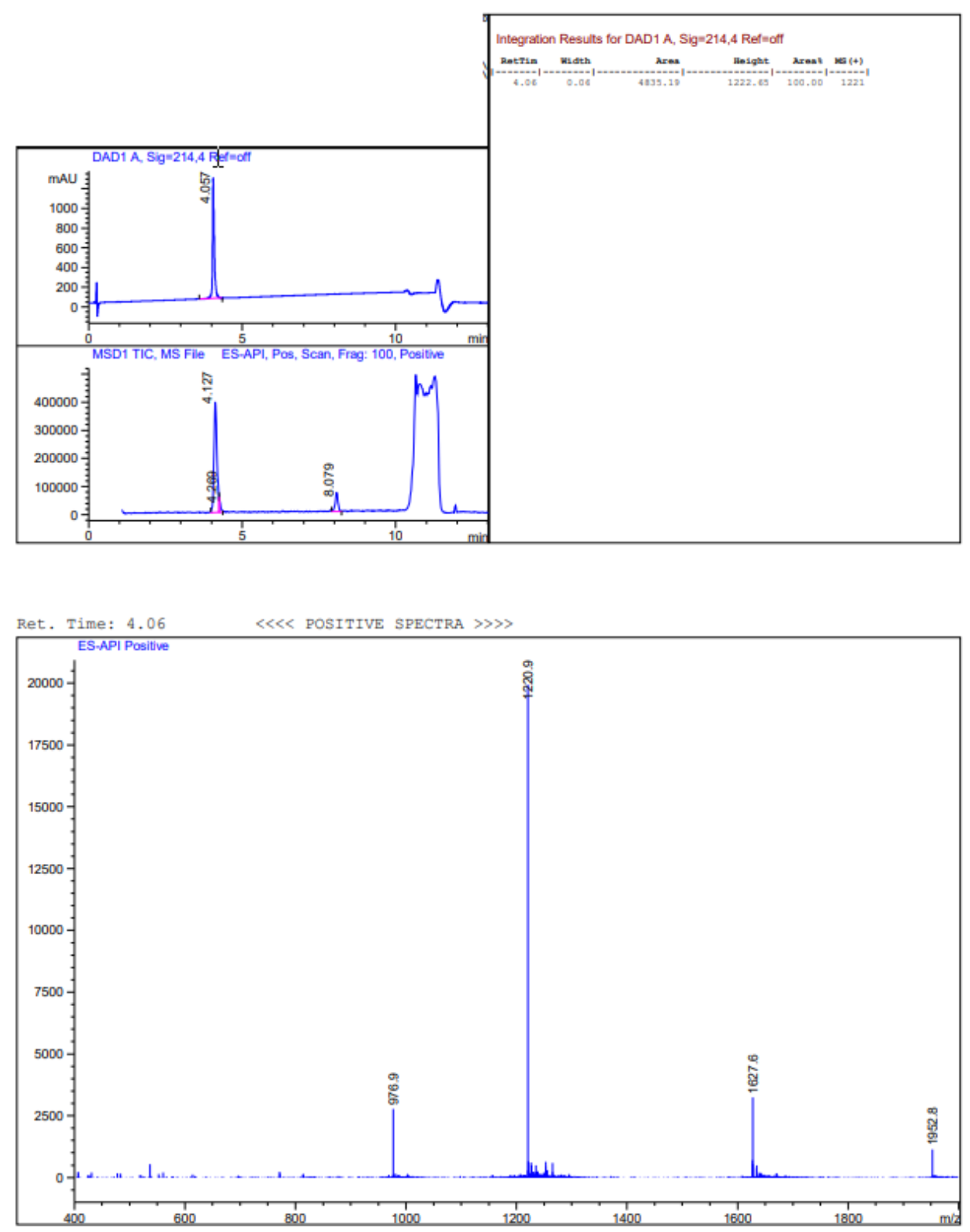
LCMS chromatogram for compound 10
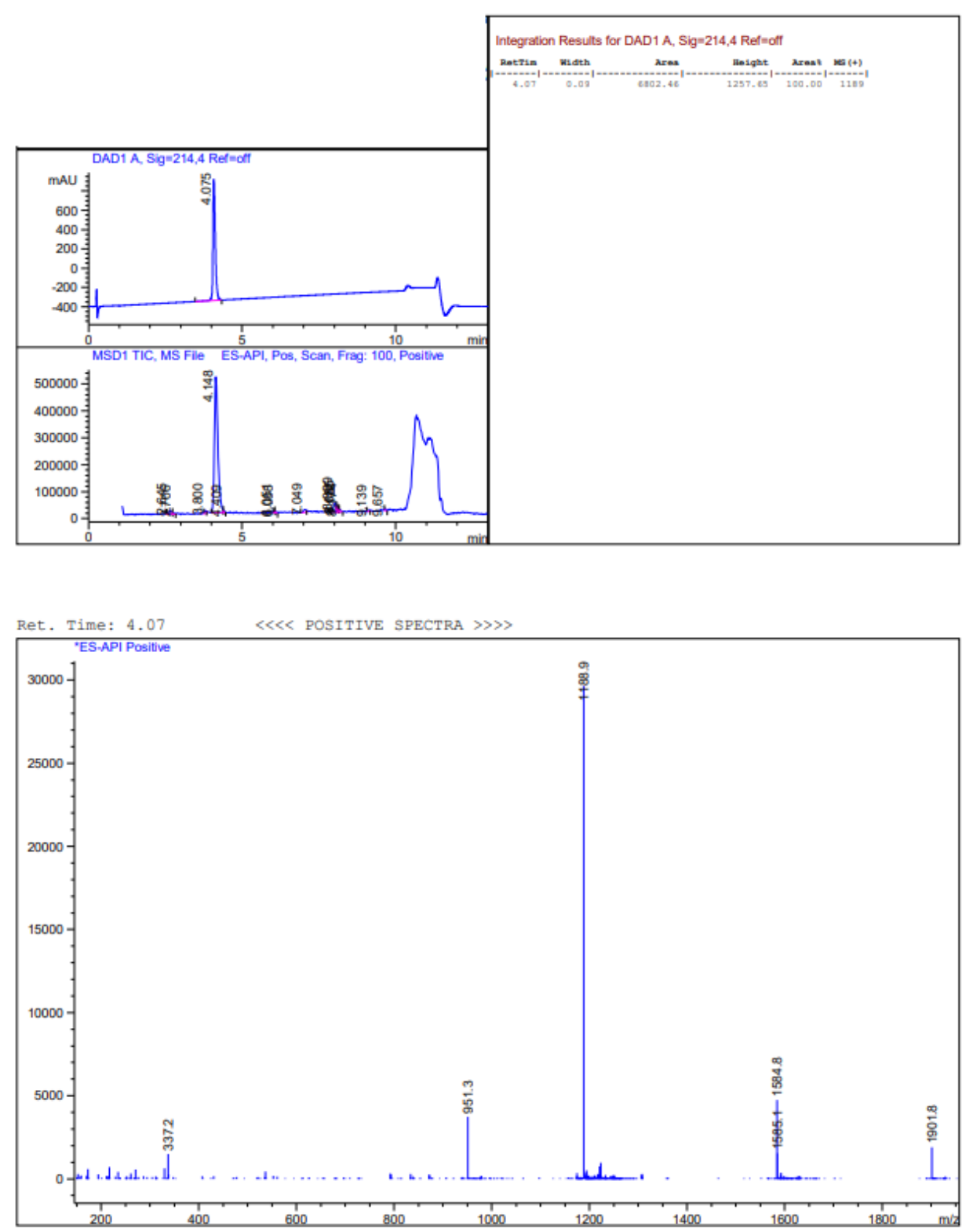
LCMS chromatogram for compound 11
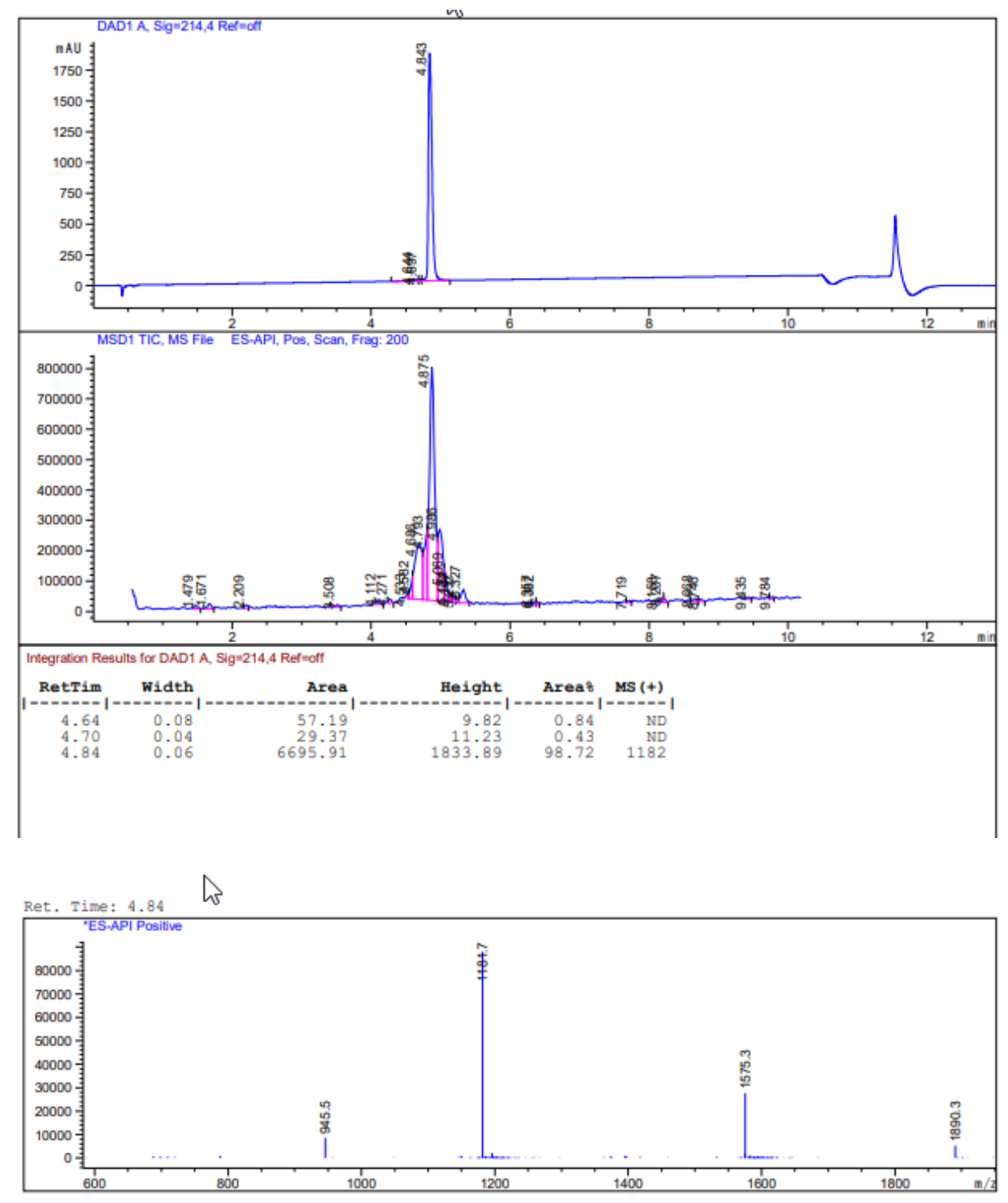
LCMS chromatogram for compound 12
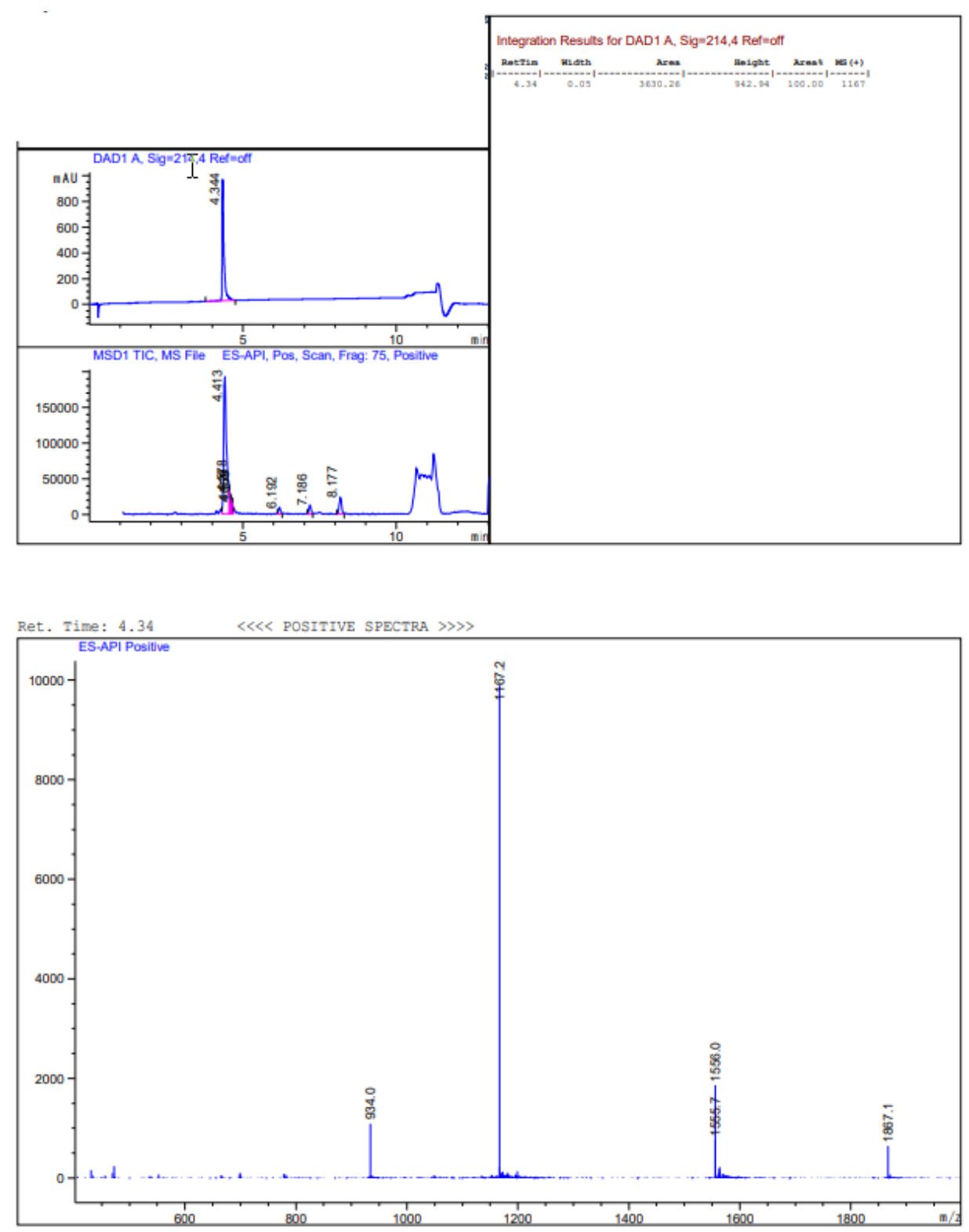

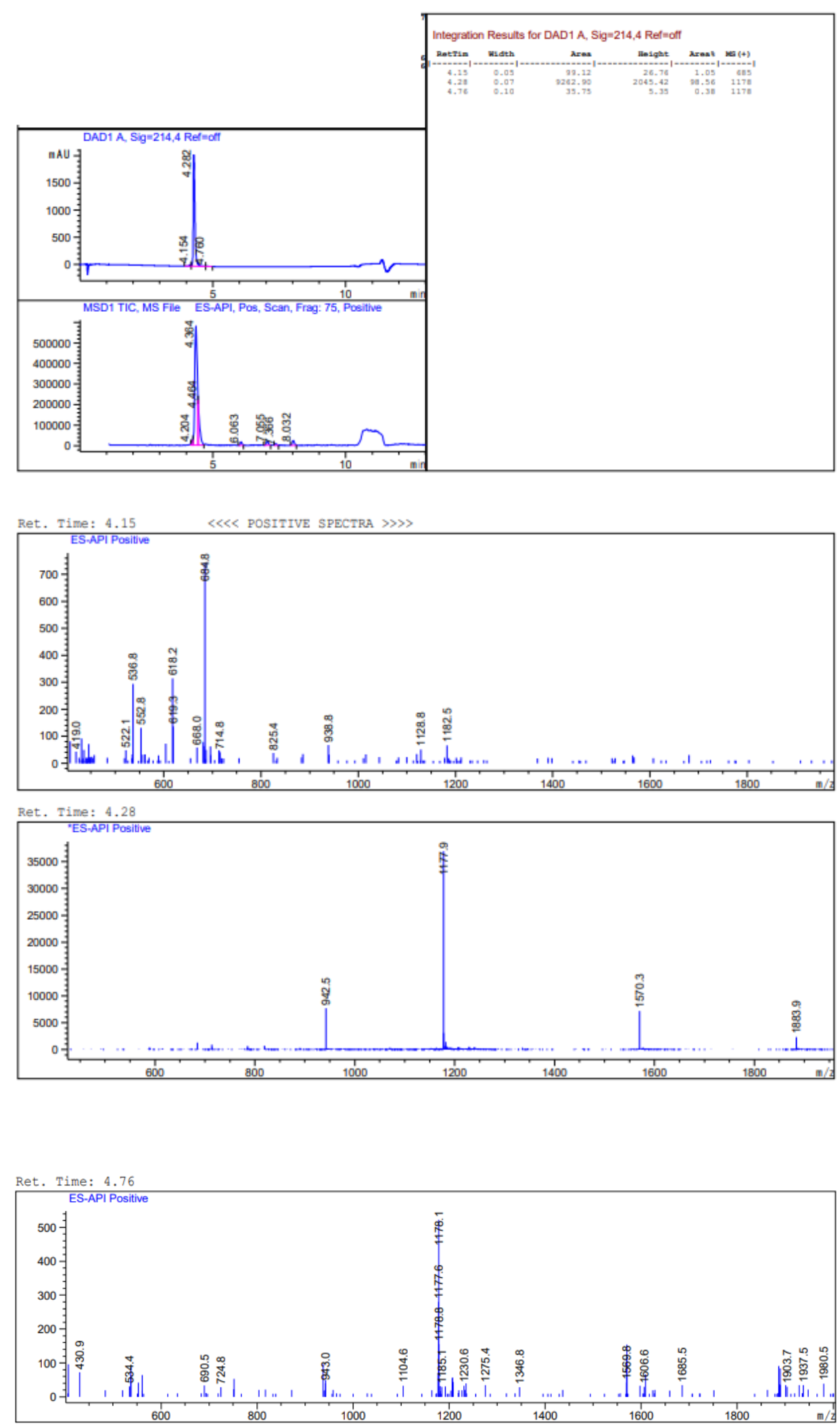

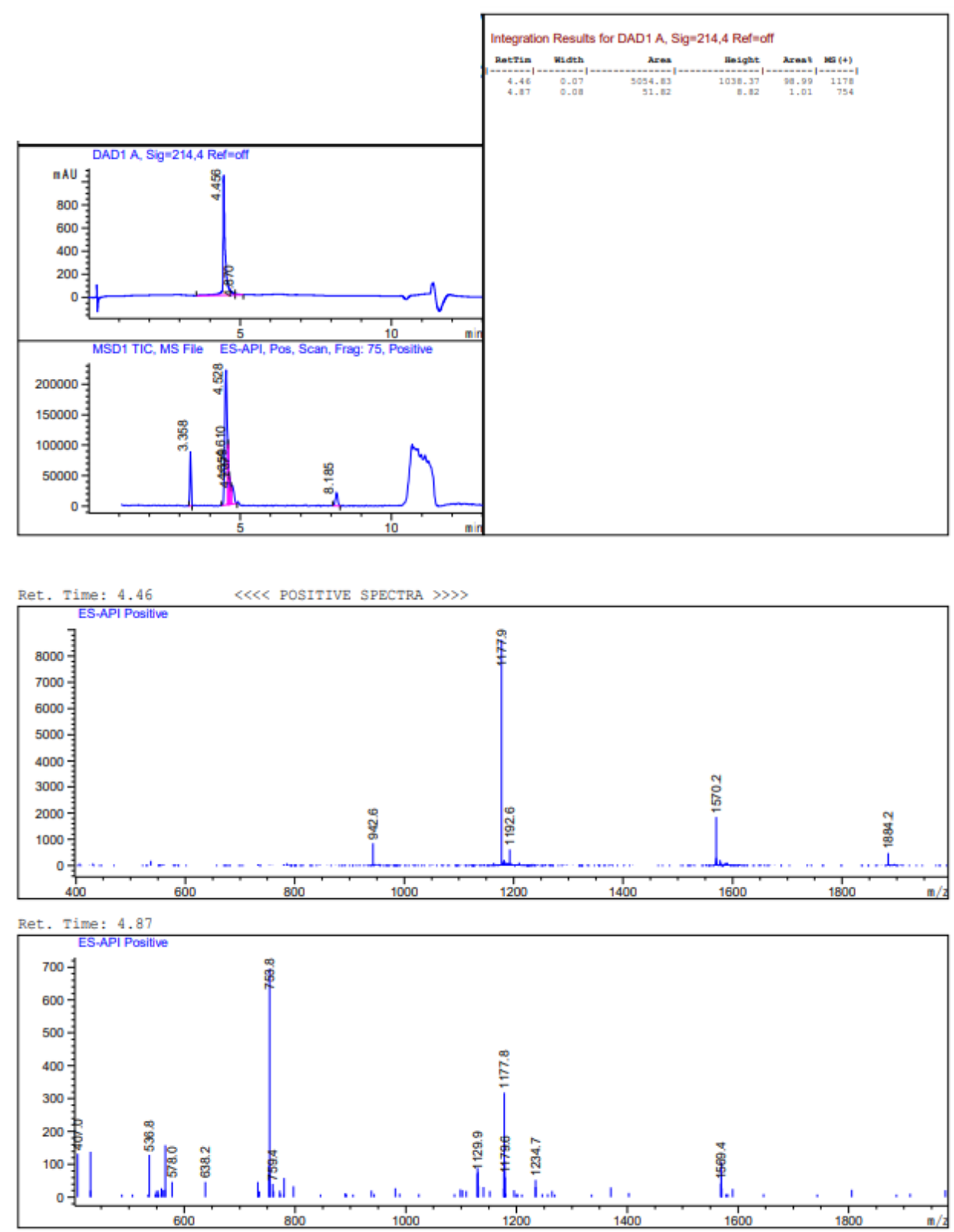
LCMS chromatogram for compound 15
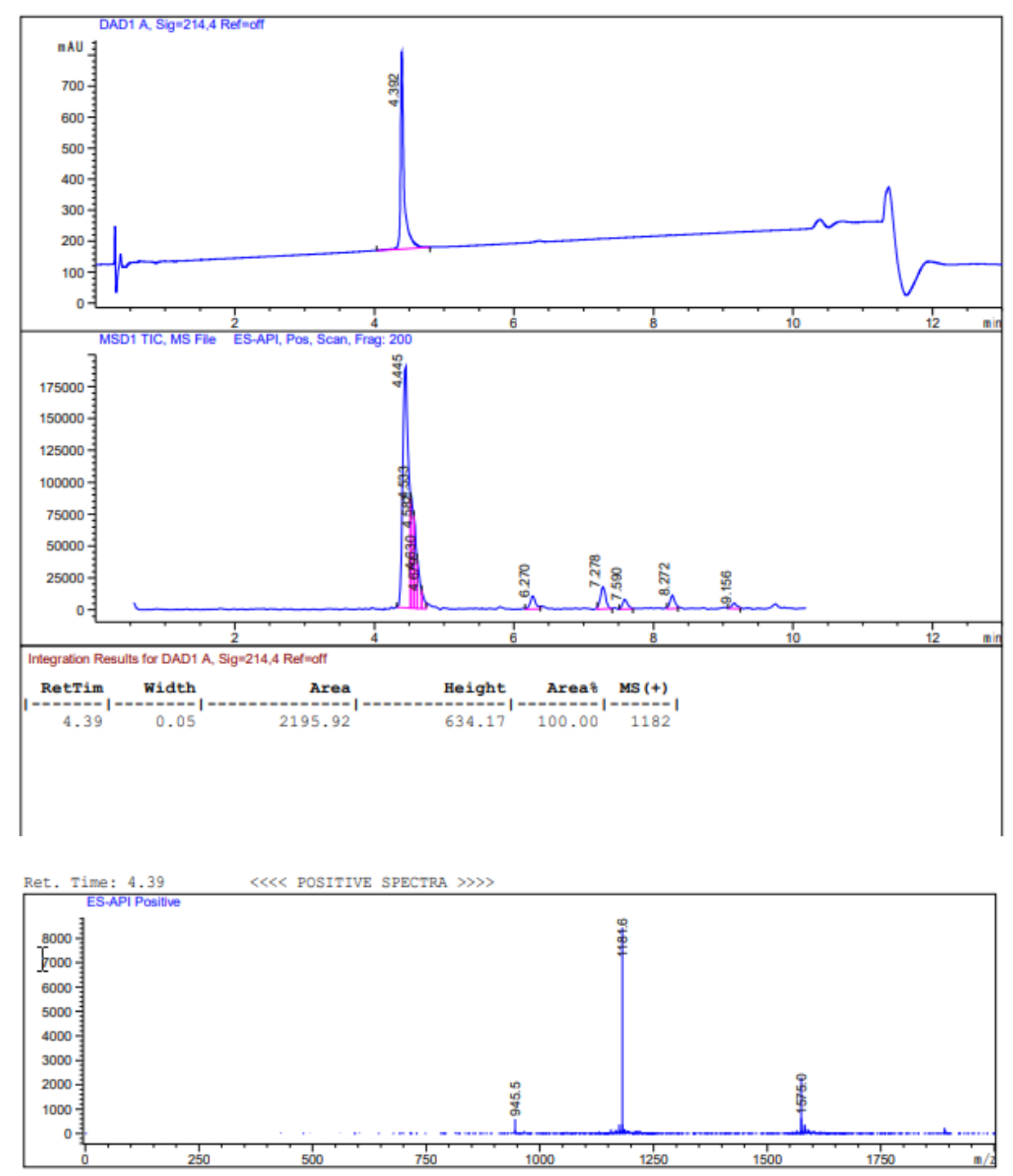
LCMS chromatogram for compound 16
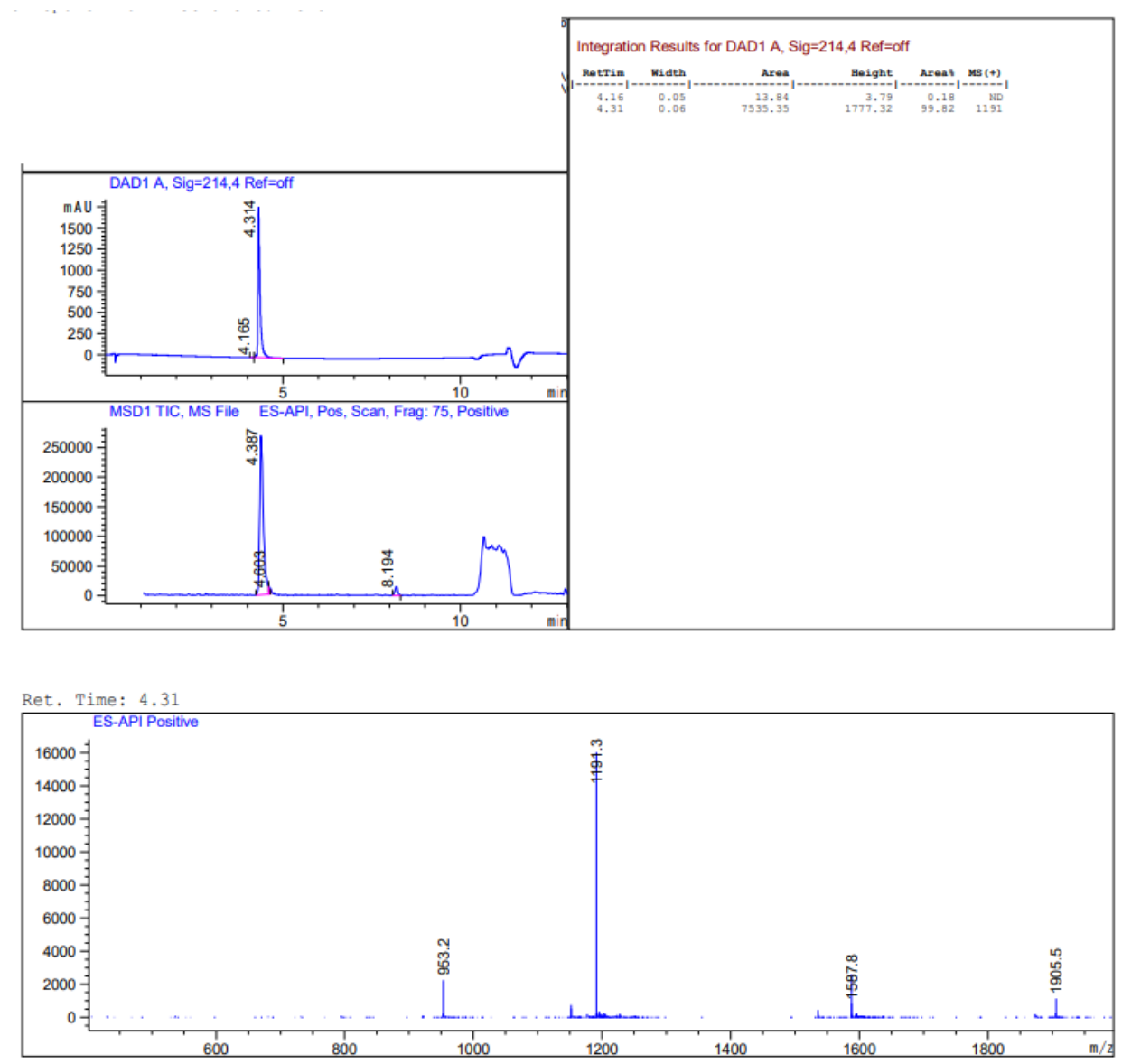
LCMS chromatogram for compound 17
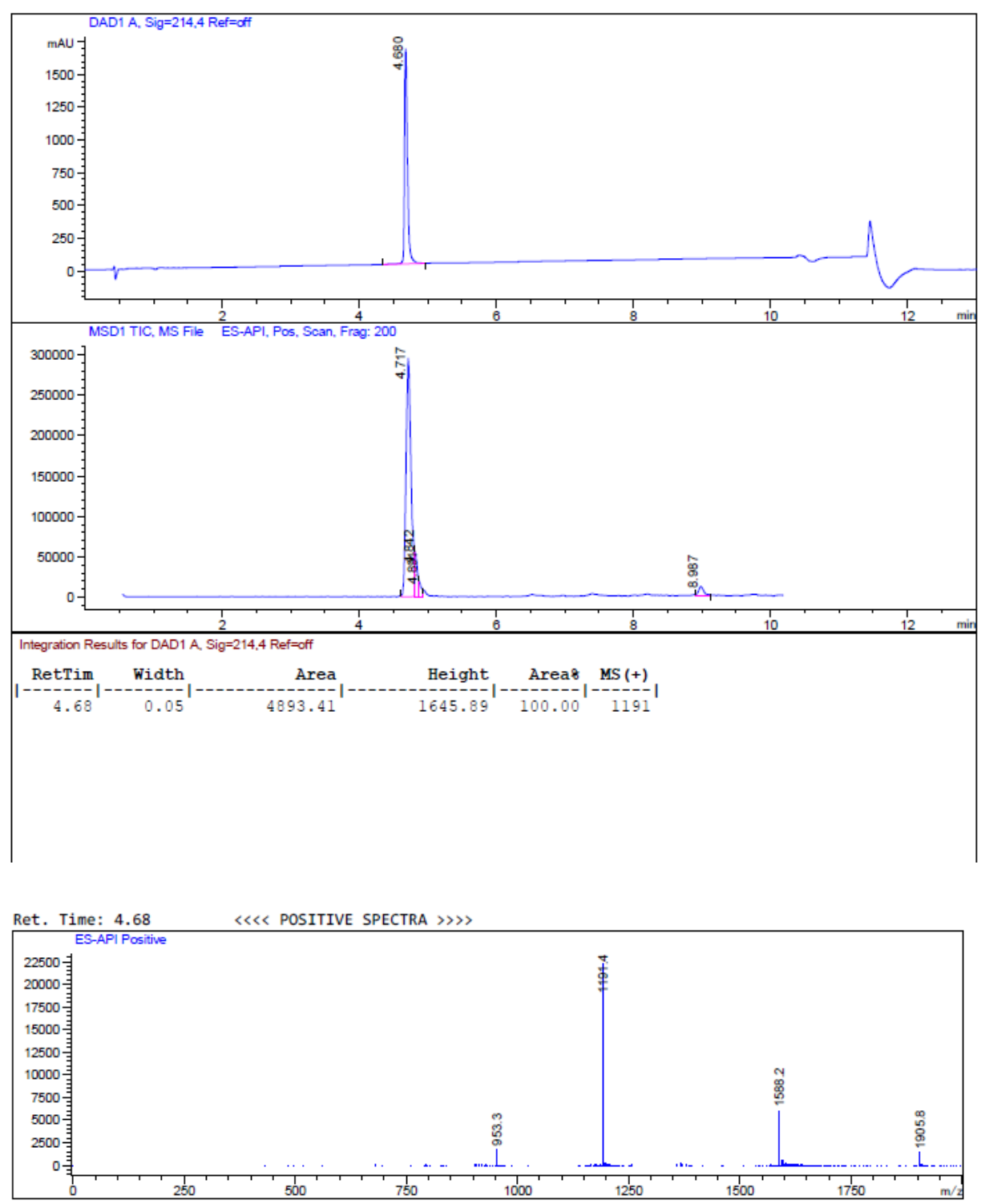
LCMS chromatogram for compound 18
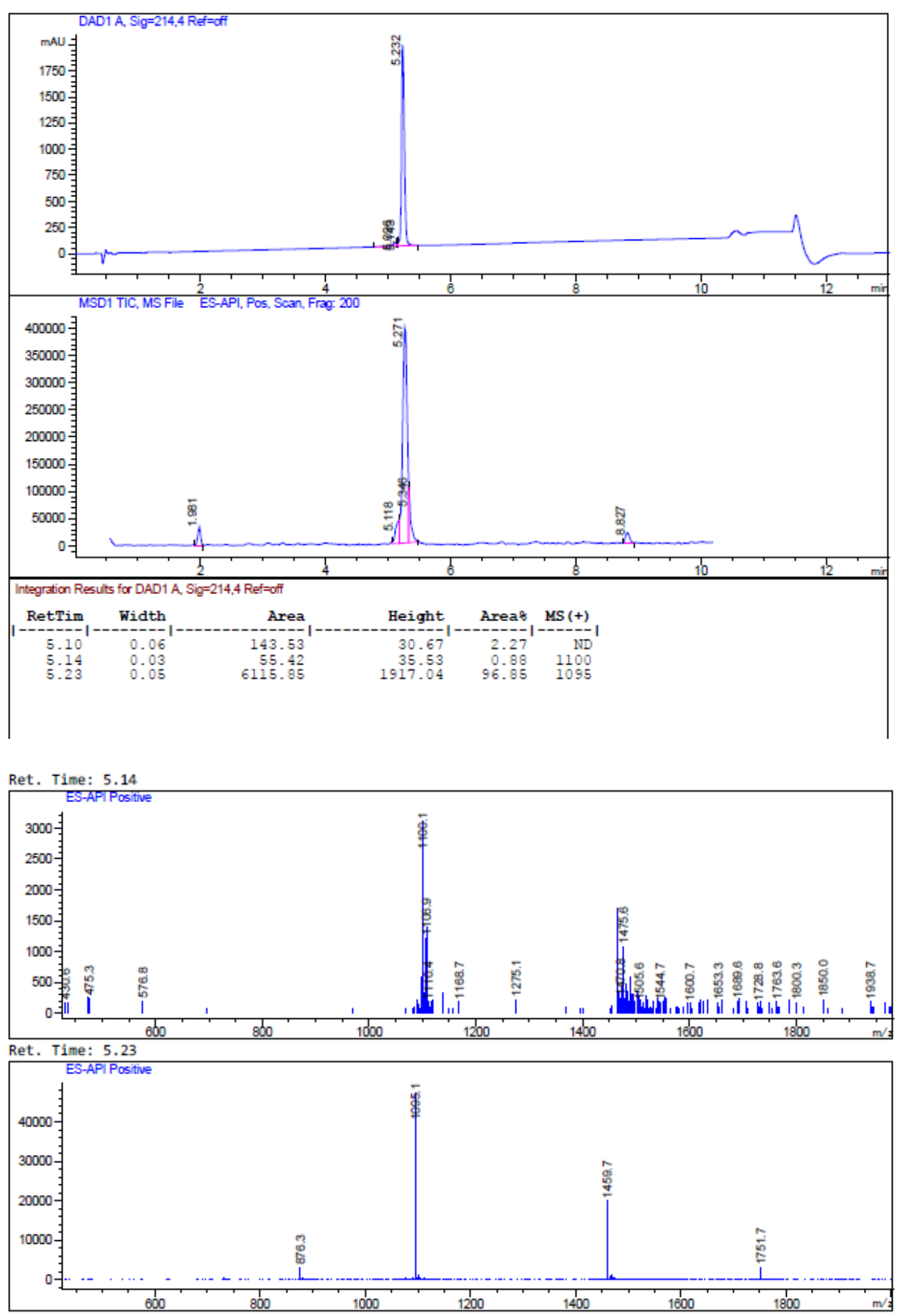
Compound Characterization Table:

\begin{tabular}{|c|c|c|c|c|c|c|}
\hline Cmpd \# & $\begin{array}{l}\text { Compound } \\
\text { description }\end{array}$ & $\begin{array}{l}H P L C \\
t_{R}(\min )\end{array}$ & $\begin{array}{r}\text { Purity } \\
\text { (\%) }\end{array}$ & $\begin{array}{r}\text { Obsd. } \\
{\left[\mathrm{M}+3 \mathrm{H}^{+}\right]^{3+}}\end{array}$ & $\begin{array}{l}\text { Calc. } \\
\text { MW }\end{array}$ & $\begin{array}{l}\text { Theor. } \\
\text { Avg. MW }\end{array}$ \\
\hline 6 & des(24-42)Maxadilan & 4.499 & 95.7 & 1670.7 & 5006.4 & 5009.71 \\
\hline 7 & $\begin{array}{r}\operatorname{des}(1-5,24- \\
\text { 42)[pE6]Maxadilan }\end{array}$ & 3.55 & 99.1 & 1126.1 & 4498.26 & 4501.14 \\
\hline 8 & $\begin{array}{r}\operatorname{des}(24- \\
\text { 42)Maxadilan(1-43) } \\
\text { acid }\end{array}$ & 4.032 & 99.8 & 1628.1 & 4878.3 & 4881.54 \\
\hline 9 & $\begin{array}{r}\operatorname{des}(24- \\
\text { 42)Maxadilan(1-42) } \\
\text { acid }\end{array}$ & 4.101 & 99.7 & 1608.9 & 4821.28 & 4824.48 \\
\hline 8 & $\begin{array}{r}\operatorname{des}(24- \\
\text { 42)Maxadilan(1-44)- } \\
\text { Amide }\end{array}$ & 3.960 & 100.0 & 1670.3 & 5005.41 & 5008.72 \\
\hline 9 & $\begin{array}{r}\operatorname{des}(24- \\
\text { 42)Maxadilan(1-43) } \\
\text { amide }\end{array}$ & 4.057 & 100.0 & 1627.6 & 4877.32 & 4880.55 \\
\hline 10 & $\begin{array}{l}\operatorname{des}(24-42,61- \\
\text { 63)Maxadilan amide }\end{array}$ & 4.075 & 100.0 & 1584.8 & 4749.26 & 4752.42 \\
\hline 11 & $\begin{array}{r}\text { [Aib50]des(24-42, } \\
\text { 45)Maxadilan(1-43) } \\
\text { amide }\end{array}$ & 4.843 & 98.7 & 1575.3 & 4719.29 & 4722.44 \\
\hline 12 & $\begin{array}{r}\text { [Aib50]des(24-42, } \\
\text { 45)Maxadilan(1-42) } \\
\text { amide }\end{array}$ & 4.314 & 100.0 & 1555.8 & 4662.27 & 4665.39 \\
\hline 13 & $\begin{array}{r}\text { [A50]des(24-42, 45, } \\
63) \text { Maxadilan(1-43) } \\
\text { amide }\end{array}$ & 4.237 & 99.5 & 1570.3 & 4705.27 & 4708.41 \\
\hline
\end{tabular}




\begin{tabular}{|c|c|c|c|c|c|c|}
\hline 14 & $\begin{array}{r}\text { a50][des(24-42, } \\
45)] \text { Maxadilan(1-43) } \\
\text { amide }\end{array}$ & 4.456 & 99.0 & 1570.2 & 4705.27 & 4708.41 \\
\hline 15 & $\begin{array}{r}\text { [Asp15;Arg16;Ala50][d } \\
\text { es(24-42, } \\
45)] \text { Maxadilan(1-43) } \\
\text { amide } \\
\end{array}$ & 4.392 & 100.0 & 1575.0 & 4720.25 & 4723.38 \\
\hline 16 & $\begin{array}{r}\text { [Ala50, Trp59, des(24- } \\
42,45,63)] \text { Maxadilan } \\
\text { amide }\end{array}$ & 4.314 & 99.8 & 1587.8 & 4758.3 & 4761.47 \\
\hline 17 & $\begin{array}{l}\text { [Asp15, Arg16, Ala50, } \\
\text { Trp59, des(24-42, 45, } \\
\text { 63)]Maxadilan amide }\end{array}$ & 4.680 & 100.0 & 1588.2 & 4759.26 & 4762.42 \\
\hline 18 & $\begin{array}{r}\text { [Ala15, Arg16, Tyr19, } \\
\text { Ala50, Trp59, des(22- } \\
45,63)] \text { Maxadilan } \\
\text { amide }\end{array}$ & 5.232 & 100.0 & 1459.7 & 4374.09 & 4377.04 \\
\hline
\end{tabular}


Data collection and refinement statistics for co-crystal structure of the PAC1R extracellular domain in complex with Peptide 18 (PDB code 7JQD)

\begin{tabular}{|c|c|}
\hline Crystal Name & PAC1R/18 \\
\hline \multicolumn{2}{|l|}{ Data Collection } \\
\hline Wavelength $(\AA)$ & 0.97741 \\
\hline Space group & P43212 \\
\hline \multicolumn{2}{|l|}{ Cell dimensions } \\
\hline $\mathrm{a}, \mathrm{b}, \mathrm{c}(\AA)$ & $67.68,67.68,56.98$ \\
\hline$\alpha, \beta, \gamma\left(\left(^{\circ}\right)\right.$ & $90,90,90$ \\
\hline Resolution $(\AA)$ & $30-2.7(2.83-2.7)^{\mathrm{a}}$ \\
\hline Completeness & 98.8 (99.9) \\
\hline Redundancy & $5.3(5.3)$ \\
\hline$R_{\text {merge }}$ & $0.2(0.904)$ \\
\hline $\mathrm{CC}(1 / 2)$ & $0.981(0.726)$ \\
\hline $\mathrm{I} / \sigma \mathrm{I}$ & $5.4(1.8)$ \\
\hline \multicolumn{2}{|l|}{ Refinement } \\
\hline Resolution $(\AA)$ & $30-2.7$ \\
\hline Complexes/ASU & 1 \\
\hline \multicolumn{2}{|l|}{ Reflections } \\
\hline Total & 3859 \\
\hline Working set & 3462 \\
\hline Test set & 397 \\
\hline $\mathrm{R}_{\mathrm{work}} / \mathrm{R}_{\text {free }}$ & $0.204 / 0.292$ \\
\hline \multicolumn{2}{|l|}{ R.m.s deviations } \\
\hline Bond lengths $(\AA)$ & 0.009 \\
\hline Bond angles $\left({ }^{\circ}\right)$ & 0.938 \\
\hline
\end{tabular}

${ }^{a}$ Numbers in parenthesis are highest resolution shell. 
Relevant SAR from alanine scanning, Aib scanning, and single residue deletion of peptide 6

\begin{tabular}{|c|c|c|c|c|c|c|c|c|c|c|c|c|c|c|c|c|c|c|c|}
\hline peptide 6 amino acids & $P$ & $G$ & $N$ & $V$ & $F$ & $K$ & $E$ & $M$ & $K$ & $Q$ & $K$ & $K$ & $K$ & $E$ & $F$ & $K$ & $A$ & $G$ & $K$ \\
\hline residue positions & $\mathbf{4 3}$ & $\mathbf{4 4}$ & $\mathbf{4 5}$ & $\mathbf{4 7}$ & $\mathbf{4 8}$ & $\mathbf{4 9}$ & $\mathbf{5 0}$ & $\mathbf{5 2}$ & $\mathbf{5 3}$ & $\mathbf{5 4}$ & $\mathbf{5 5}$ & $\mathbf{5 6}$ & $\mathbf{5 7}$ & $\mathbf{5 8}$ & $\mathbf{5 9}$ & $\mathbf{6 0}$ & $\mathbf{6 1}$ & $\mathbf{6 2}$ & $\mathbf{6 3}$ \\
\hline Ala scanning & & & & & & & & & & & & & & & & & & & \\
\hline Aib scanning & & & & & & & & & & & & & & & & & & & \\
\hline single residue deletions & & & & & & & & & & & & & & & & & & & \\
\hline
\end{tabular}

$>2 x$ potency improvement

$<10 x$ potency loss

between $10 x-100 x$ potency loss

no activity observed at 1000nM

\section{Additional information on commercial M65 reference compound tested:}

Source: Bachem

Name: M65 trifluoroacetate salt / PAC1 receptor antagonist M65

Catalog number: $\mathrm{H}-6736$

Sequence comparison

C(1)DATC(1)QFRKAIDDC(2)QKQAHHSNVPGNSVFKEC(2)MKQKKEFKA(amide C-term) (commercial M65) C(1)DATC(1)QFRKAIDDC(2)QKQAHHSNVLLPGNSVFKEC(2)MKQKKKEFKA (M65, 5; sequence from reference \#18) C(1)DATC(1)QFRKAIDD C(2)QKQAHHSNVPGNSVFKEC(2)MKQKKKEFKAGK (Max.d.4 or des(24-42)Maxadilan, 6)

*underlined $\mathrm{C}$ denotes presence of Cys $(\mathrm{x})$-Cys $(\mathrm{x})$ disulfide bond

Table 2. PAC1R Activity of Reported Antagonists

\begin{tabular}{rccc}
\hline & $\begin{array}{c}\text { hPAC1R IC } 50 \\
\text { (nM) }\end{array}$ & $\begin{array}{c}\text { hVPAC1 IC } 50 \\
\text { (nM) }\end{array}$ & $\begin{array}{c}\text { hVPAC2 IC } 50 \\
\text { (nM) }\end{array}$ \\
\hline PACAP(6-38) (4) & 3.5 & 118.4 & 4.5 \\
Commercial M65 & $>100000$ & & \\
des(24-42)Maxadilan (6) & $11 \pm 3.5$ & $>20000$ & $>20000$ \\
$\operatorname{des}(1-5,24-42)[p E 6]$ Maxadilan (7) & 3211 & $>20000$ & $>20000$ \\
\hline
\end{tabular}

*Assay measures inhibition of PAC1R after challenge with PAC1R agonist PACAP38. 